\title{
Design and Implementation of a Harmonic Monitoring Device Based on STM32
}

\author{
Yong Song ${ }^{1, a^{*}}$ and Yixin $\mathrm{Su}^{2, \mathrm{~b}}$ \\ ${ }^{1}$ School of Automation, Wuhan University of Technology, Wuhan, Hubei ,430000, China \\ ${ }^{2}$ School of Automation, Wuhan University of Technology, Wuhan, Hubei ,430000, China \\ a2452943055@qq.com, b937340186@qq.com
}

\begin{abstract}
Keywords: harmonic monitoring, STM32F103, ADE7878, signal sampling
Abstract. Monitoring harmonic in power grid is particularly important because harmonic will seriously affect the quality of power energy. Based on a high-performance 32-bit processor STM32F103 and a electric energy metering chip ADE7878, a harmonic monitoring device is designed for measuring 2-31 harmonics in the grid. Firstly, the function, the overall architecture and the working principle of the harmonic monitoring device are introduced. Then the system hardware schematic diagram is analyzed and the flow chart of software is given. The results of application show that the harmonic monitoring device can accurately measure 2-31 harmonic in the grid .
\end{abstract}

\section{Introduction}

With the improvement of living standards, the demand for electricity is growing and more and more high quality for electricity is required. But the use of a large amount of non-linear load has produced plenty of harmonics, which does great harm to the grid $^{[1]}$, influencing the power quality. Harmonic monitoring as the most basic work is very important. Only accurately grasp the grid information can we quickly find out the problem ,put forward the corresponding solutions and obtain the high quality of electricity.

Traditional harmonic monitor with the single chip processor as the core is unable to meet the needs of the current power system because of the low measuring accuracy.the harmonic monitor based on DSP chip is difficult to promote on account of the high cost ${ }^{[2]}$. In view of the current status quo, this paper designed a harmonic monitor based on STM32F103 and ADE7878 so that we can detect harmonic in grid accurately. The harmonic monitor has the advantages of simple, reliable, high precision and low cost.

\section{System Function and Overall Design}

The harmonic monitor can analyze 2-31 harmonic for their amplitude, phase and total harmonic distortion rate, every harmonic component of RMS, power factor for phase and other major parameters. These parameters can be separately display on the LCD screen, and we can give an alarm if the harmonic crosses the line. The harmonic monitor have communication interface so that we can transmit data to the upper computer for monitoring harmonic through the Ethernet. The upper computer is based on WINDOWS operating system, including the functions of receiving data, storage of data, database management, harmonic dynamic monitoring and analysis. It can collect various parameters to generate the corresponding reports, graphs and pie charts, histograms, etc.. At the same time it can provide harmonic dynamic monitoring and analysis result, and gives the corresponding guidance. In addition, the harmonic monitor provides query function, and we can print the output.

The system adopts three-phase multifunction metering chip ADE7878 and microcontrollers STM32F103 as the main architecture. Metering chip completes the measurement of all kinds of electric parameter ${ }^{[3]}$. Micro controller completes the task of data analysis, processing, display, storage, control and communications, etc..

The structure of harmonic monitor is shown in Fig. 1. The system is mainly composed of the minimum system of STM32F103, sampling circuit for voltage and current, measurement circuit for the electric parameters, communication circuit, alarm circuit, key, LCD, clock circuit and power supply. 


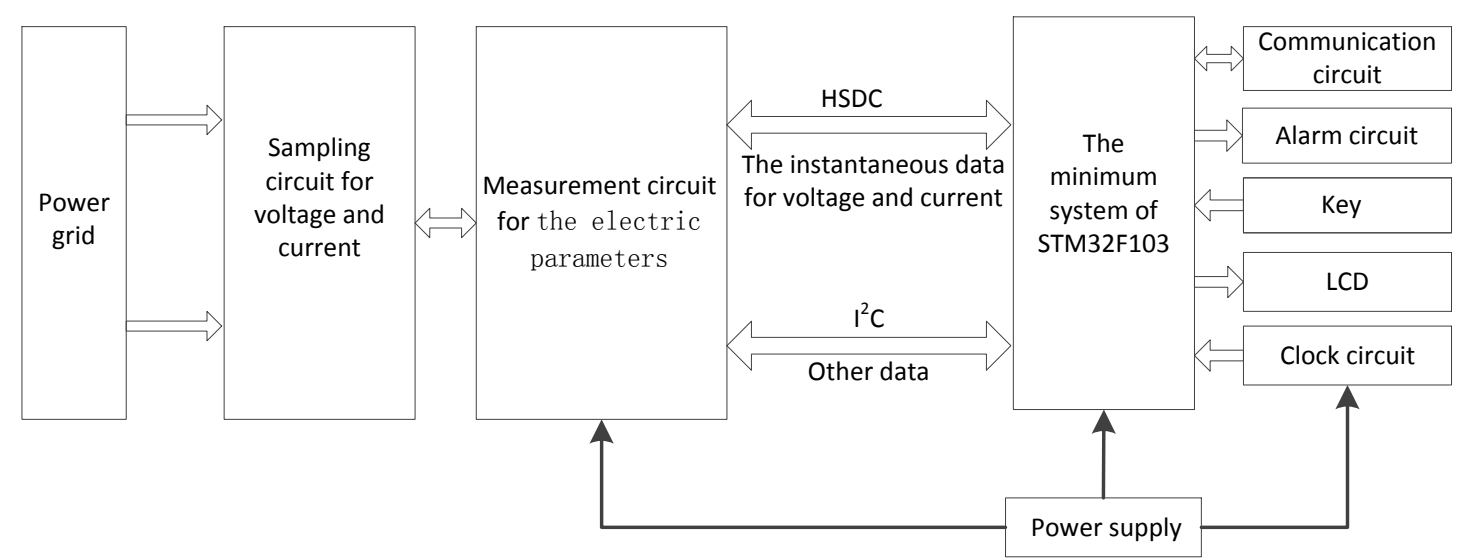

Fig. 1. The structure diagram of harmonic monitor

The Fig. 1 shows that the $220 \mathrm{~V}$ voltage in grid is converted into $0.5 \mathrm{~V}$ through voltage and current sampling circuit to meet the demand of the metering chip ADE7878; ADE7878 transmit the information of the phase voltage, current and other parameters to STM32F103; STM32F103 calculates the corresponding harmonic parameters by the related algorithm of harmonic analysis, and the related data is displayed on the LCD. In addition, the Ethernet communication module is also designed for the remote transmission of harmonic data.

\section{The Design of System Hardware}

Signal sampling circuit includes voltage transformer (PT), current transformer (CT) and filtering.It can change large current into a standard voltage signal that can be input into metering chip ${ }^{[4]}$. The A phase current, voltage principle diagram is shown in Fig. 2, Fig. 3 respectively. The output current of CT in the current input channel, is changed into a voltage signal through the resistor sample.Then it is input into ADE7878 through filtering capacitor filters. Without this filtering capacitor, the part that input frequency is higher than two times of the sampling frequency will stack in the low frequency part,then the measured values of the low frequency component is higher than the actual value. Voltage input circuit consists of voltage transformer and RC filtering network. Compared with partial pressure resistance circuit, the PT circuit can not only simplify the circuit,but also achieve the isolation of measurement circuit and the grid. The stand or fall of voltage and current sampling circuit will directly affect the accuracy of measurement.

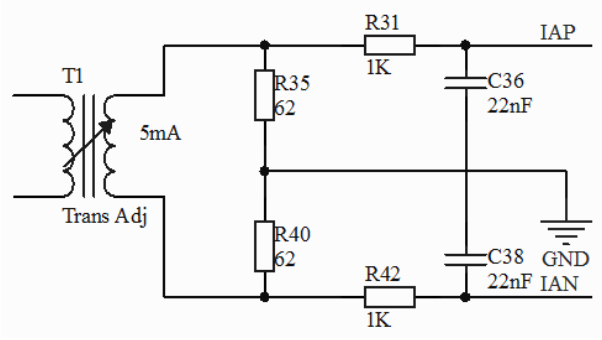

Fig. 2. A phase current input schematic diagram

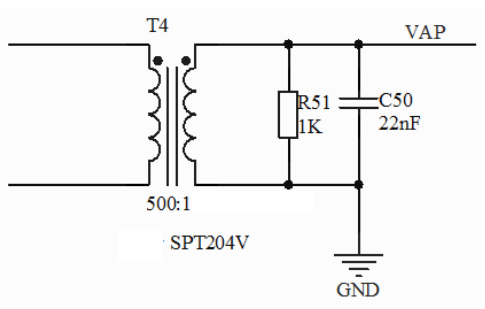

Fig. 3. A phase voltage input principle diagram

The latest ADI metering chip ADE7878 is used for the processing of sampling voltage and current signal. ADE7878 interface circuit is shown in Fig. 4. The chip can measure current/voltage effective value, total (fundamental wave and harmonic) active power, reactive power and apparent power, fundamental active power and reactive power. At the same time,it can also provide the active and reactive power (fundamental wave and harmonic), total energy, the fundamental active and non function, while the harmonic energy can be obtained by simple subtraction operation.

System's core is STM32F103, which can perform the harmonic analysis, peripheral chip control, keyboard input, data display, storage and communication, etc.It communicates with the ADE7878 by the way of I2C and HSDC.I2C is a two-wire serial bus. HSDC is a custom protocol that works on the SPI interface.It allows ADE7878 automatically to send electric parameters of instantaneous value to STM32F103. For a period of time carry on the fast Fourier transform for the voltage and current sampling sequence.The interface between STM32F103 and ADE7878 is shown in Fig. 4. 


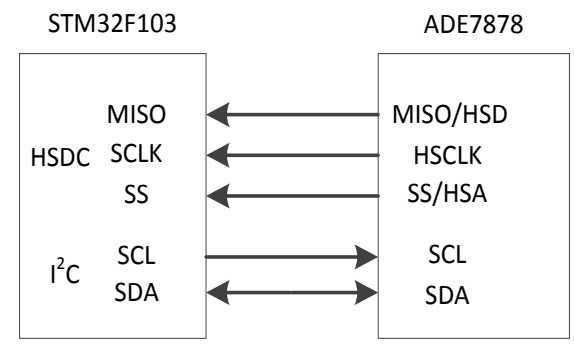

Fig. 4. The interface between STM32F103 and ADE7878

\section{The Design of System Software}

The main job of STM32F103 is to periodically read the instantaneous value of voltage, current and power, carry out FFT algorithm for harmonic parameters and refresh the LCD display. Because the FFT operation needs a complete cycle of fundamental wave, sample in the program of interrupt service on a regular basis. According to the actual needs, sample 160 points in a cycle, namely every $0.125 \mathrm{~ms}$ read HSDC port high-speed transmission of sampling data. After read a complete cycle of data,perform quickly FFT algorithm for harmonic parameter in the next $20 \mathrm{~ms}$ and complete the work of refreshing LCD. System of the main program and interrupt program flow chart are shown in Fig. 5, Fig. 6.

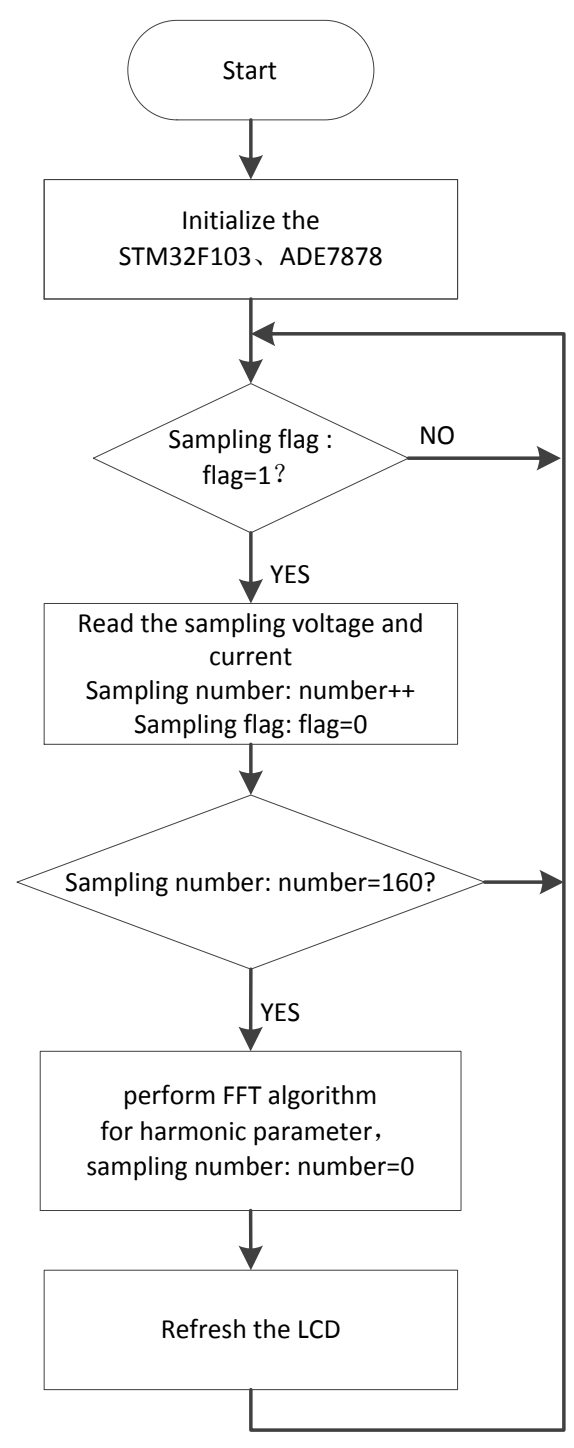

Fig. 5 main program flow chart

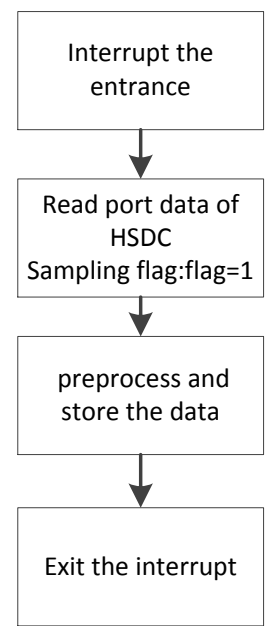

Fig. 6 interrupt program flow chart 


\section{Harmonic Analysis Software}

The upper computer of harmonic analysis software which is based on WINDOWS operating system, is programed by VC.The upper computer of harmonic analysis software mainly includes receiving data, storage, database management, harmonic dynamic monitoring, analysis and display and other functions. It can collect various parameters to generate the corresponding reports, graphs and pie charts, histograms, etc.. At the same time it can provide harmonic dynamic monitoring and analysis result, and gives the corresponding guidance. In addition, the harmonic monitor provides query function, and we can print the output.

\section{Conclusion}

In this paper, a harmonic monitor based on the high performance processing chip STM32F103 and metering chip ADE7878 is designed. The high precision metering chip ADE7878 in the harmonic monitor is used to obtain each phase voltage, current, power value from the sampling voltage and current signal. STM32F103 perform FFT arithmetic to calculate the harmonic parameter and save the data after each phase voltage, current value and the corresponding phase information are obtained. The user can query each phase voltage, current and harmonic parameters by using keys. The design can meet the demand of measuring and monitoring harmonic. At the same time, it has the advantage of simpleness, reliability, stability and low cost, which is beneficial to marketing.

\section{References}

[1] Santoso S,Grady W M,Edward J,et al.Characterization of distribution power quality events with Fourier and wavelet transforms. IEEE Transactions on Power Delivery . 2000.

[2] Xiao M Y,Xiao H L.The harmonic detection based on wavelet transform and FFT for electric arc furnaces. Proceedings of the 2009 International Conference on Wavelet Analysis and Pattern Recognition ,2009.

[3] Ahmet S. Yilmaz,Ahmet Alkan,Musa H. Asyali. Applications of parametric spectral estimation methods on detection of power system harmonics[J]. Electric Power Systems Research. $2007,11(4): 75-79$.

[4] Julio Barros, Ramón I. Diego. A new method for measurement of harmonic groups in power systems using wavelet analysis in the IEC standard framework[J]. Electric Power Systems Research,2005,23(4):104-121. 